\title{
Using Proseal LMA and I-gel for difficult airway management in patient with diffuse tracheal stenosis and pulmonary artery sling
}

\author{
Kosucu $\mathrm{M}^{1}$, Eroglu A ${ }^{1}$, Besir $\mathrm{A}^{2}$, Cansu $\mathrm{A}^{3}$ \\ Karadeniz Technical University, Department of Anesthesiology, Trabzon, Turkey. mugekk73@hotmail.com
}

\begin{abstract}
Pulmonary artery sling (PAS) is a rare congenital anomaly frequently accompanied by focal or longsegment tracheal stenosis. We presented a 34-year-old female patient with severe long-segment tracheal stenosis associated with PAS which was assessed with Multi Slice Computed Tomography (MSCT) consisting of axial, three dimensional and virtual bronchoscopic images of the airway. This case report discussed the management of her difficult airway condition during surgical cholecystectomy and thyroidectomy by using proseal- LMA and I-gel, respectively (Fig. 4, Ref. 10). Full Text in PDF www.elis.sk.

Key words: pulmonary artery sling, tracheal stenosis, proseal-LMA, I-gel.
\end{abstract}

Pulmonary artery sling (PAS) is an extremely rare congenital heart anomaly. PAS is described as a condition when the left pulmonary artery arises from the right pulmonary artery while forming a sling around the trachea and causing tracheal compression (1). In $55 \%$ to $60 \%$ of patients, the tracheal rings are complete to various extents with intrinsic tracheal stenosis (TS) (2). Complete tracheal rings presenting in adulthood are extremely rare (3).

Unexpected difficulties or failures in airway management are still important factors in morbidity and mortality related to anesthesia. A careful preoperative evaluation of the airways and a carefully considered plan for intubation are the most important issues in patients with abnormal airways. We described a 34-yearold female patient with severe long-segment TS associated with PAS which was assessed with multi-slice computed tomography (MSCT) of her airway. In this case, we reported the management of the difficult airway condition during surgical cholecystectomy and thyroidectomy by using proseal-LMA and I-gel, respectively.

\section{Case report}

A 34-year-old female, with ASA physical status classification II, weighing $66 \mathrm{~kg}$, with a history of unsuccessful intubation was scheduled for cholecystectomy. The patient was transferred to our hospital after her surgery being postponed due to unsuccessful intubation. The reason for unsuccessful intubation was that the intubation tube could not be forwarded. She had a benign multinodular goiter. She had no significant previous medical history and had no abnormal finding on preoperative biochemical testing, including

${ }^{1}$ Karadeniz Technical University, Department of Anesthesiology, Trabzon, Turkey, ${ }^{2}$ Staff of Anesthesiologist, Trabzon, Turkey, and ${ }^{3}$ Karadeniz Technical University, Department of Radiology, Trabzon, Turkey

Address for correspondence: M. Kosucu, MD, Department of Anesthesiology, KTU Farabi Hospital, 61080 Trabzon, Turkey.

Phone: +90.4623775904, Fax: +90.4623250518

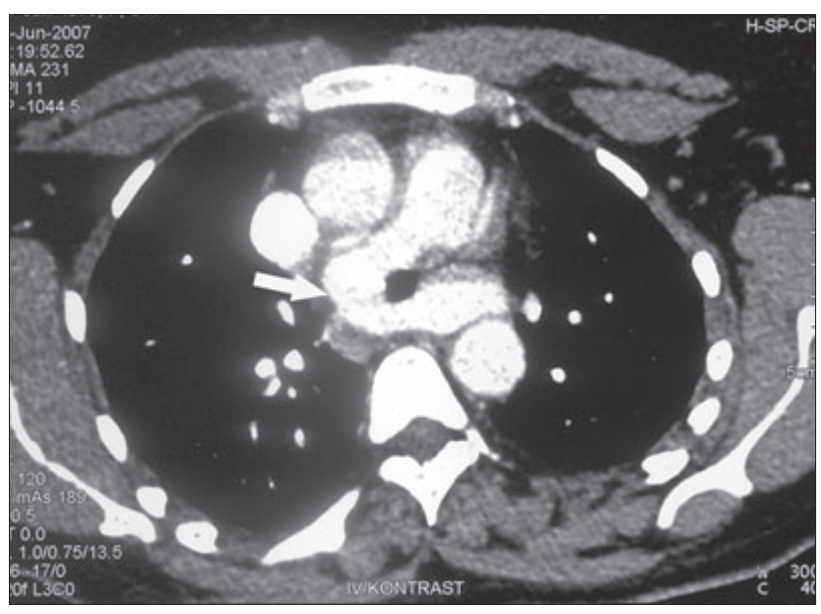

Fig. 1. Axial CT scan shows anomalous left pulmonary artery arising from the right pulmonary artery and compressing the trachea.

thyroid function tests. The assessment of her airway revealed a Mallampati score of 2, thyromental distance of $7 \mathrm{~cm}$, sternomental distance of $14 \mathrm{~cm}$ and interincisor gap of $5 \mathrm{~cm}$, all of which were normal. Preoperative chest $\mathrm{x}$-ray showed a tracheal deviation to the right. Thereafter, a radiologist performed MSCT to obtain 3D figures of the trachea. MSCT axial and 3D volume-rendered angiographic reconstruction images showed PAS. The pulmonary trunk of normal size continued rightward as did the proximal right pulmonary artery. The left pulmonary artery was arising from the right pulmonary artery while forming a vascular sling compression of the trachea (Fig. 1). The axial images, 3-D reconstruction and virtual bronchoscopic imaging of the tracheobronchial tree demonstrated a long segment of carrot-shaped irregular tracheal stenosis with its smaller diameter reaching the proximal trachea (Figs 2, 3). MSCT examinations revealed a tracheal stenosis beginning 3 $\mathrm{cm}$ below the glottis. The diameter of the stenotic portion of the trachea was $5 \mathrm{~mm}$. There was also a proximal tracheal distortion 


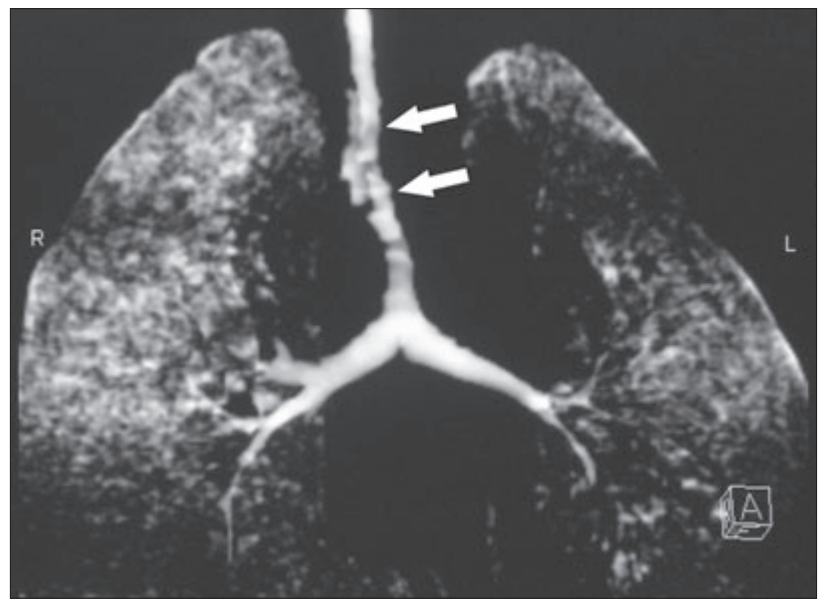

Fig. 2. Coronal 3DCT shows a long-segment tracheal stenosis.

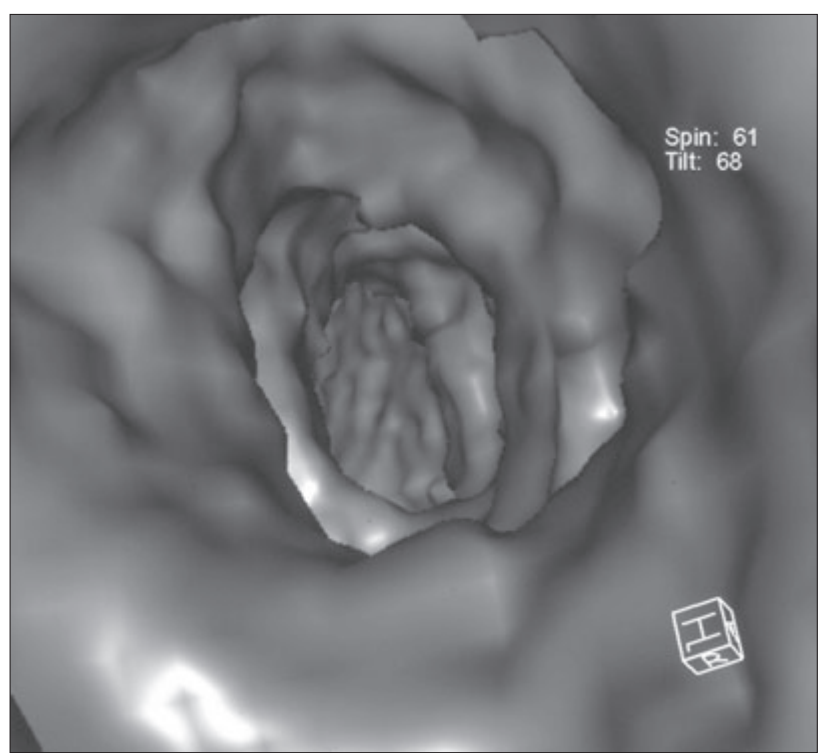

Fig. 3. Virtual bronchoscopy image shows stenosis and irregularity in the inner surface of the trachea.

to the right due to a large nodule at the left thyroid lobe (Fig. 4).

During her preoperative visit, on physical examination, the patient did not have dyspnea, inspiratory stridor or any other respiratory complaint. Her respiratory function tests decreased minimally (vital capacity $76 \%$ of expected, $\mathrm{FEV}_{1} 77 \%$ ). In the operation theatre, standard monitors including electrocardiography, automated non-invasive blood pressure, pulse oximeter, capnometer, pericardial stethoscope, nasopharyngeal temperature, and emergency tracheotomy were available. General anaesthesia was induced with IV midazolam $2 \mathrm{mg}$, IV pentothal $400 \mathrm{mg}$ and IV remifentanyl $70 \mathrm{mg}$. Mask ventilation was quite satisfactory throughout spontaneous breathing. We initially placed a size-4 Proseal-Laryngeal Mask Airway (P-LMA) without muscle relaxation, and the placement was successful on first attempt. Thereafter, the mechanical ventilation was started in volume-controlled mode by $6-8 \%$ desflourane, mixture of $\mathrm{O}_{2}-\mathrm{N}_{2} \mathrm{O} 40-60 \%$ and

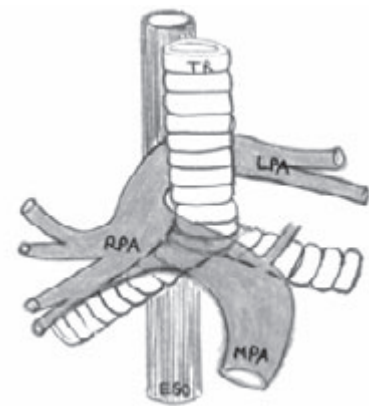

Fig. 4. Illustration of pulmonary artery sling (PAS) (RPA - right pulmonary artery, LPA - left pulmonary artery, MPA - main pulmonary artery, ESO - esophagus, TR - trachea).

neuromuscular blockade. Ventilation was set to $8 \mathrm{~mL}^{-1} / \mathrm{kg}^{-1}$ with PEEP of $5 \mathrm{~cm} \mathrm{H}_{2} \mathrm{O}$. The respiratory rate was adjusted to maintain end-tidal $\mathrm{CO}_{2}$ between $35-45 \mathrm{mmHg}$. Hemodynamic parameters, peak inspiratory pressure and end-tidal $\mathrm{CO}_{2}$ were noted at different time points. The operation ended, proseal LMA was removed and the patient was woken up without any problem.

About one month later, the patient was admitted to hospital for surgical thyroidectomy. After the same kind of preparations for the operation theatre, the emergency tracheotomy conditions were again available. She was given general anesthesia by using the same kind of drugs. I-gel was inserted this time, and both insertion and ventilation were successfully performed on first attempt without any difficulty. The operation lasted 110 minutes, and I-gel was removed without any problem.

\section{Discussion}

Difficulties or failures in airway management are still important factors in morbidity and mortality related to anesthesia. TS is one of the causes of difficulties or failures in the airway management. Recommended methods to resolve a critical airway event include a laryngeal mask airway (LMA), transtracheal jet ventilation (SMFJV), esophageal tracheal combitube (ETCT), or surgical airway to avoid potential morbidity and mortality according to ASA (4). In our case, the narrowest point of the trachea, starting from 3 $\mathrm{cm}$ below the vocal cord, was $5 \mathrm{~mm}$ in diameter as seen on MSCT, with a stenosis which held the whole trachea. We think that a very small size ET, for example 3.5 or 4 French can be accessed from this hole. But it could be very dangerous as to some unwanted side effects. If the patient had been intubated, the airway pressure could have risen and ventilation could have become difficult due to the small-calibre intubation tube. We did not attempt to perform any fiberoptic intubation due to similar reasons. Forced insertion of ET or repeated attempts to do so could damage the trachea due to stenosis. Perforation, bleeding, and shearing of the stenotic tracheal tissue may induce a loss of airway control.

The ETCT is a double-lumen airway device for providing either supraglottic or tracheal ventilation. It has high success rates with minimal trauma in surgical or ICU patients. It is a good alternative airway for managing the "cannot-intubate-cannot-ventilate" 
situations while ASA supports this idea because its insertion may be possible from affront without visual control and with head and neck in neutral position (5). It has been suggested for emergency ventilation with success rates of about $90 \%$, namely after failed tracheal intubation (6).

The SMFJV technique has generally been preferred during TS surgical intervention in order to control the airway in literature; nevertheless, for our patient it could have been more than an invasive approach. It would be used to ventilate the patients, should the stenosis of the airway be involved in surgical procedure (7).

The LMA has been extensively used for the administration of general anesthesia. It may be more useful for rigid infraglottic tracheal pathologies. Unlike ET, LMA or P-LMA, and I-gel cannot bypass the laryngotracheal pathology. They offer the control of the airway without intubating the diffuse stenotic trachea. In our opinion, supraglottic ventilation via LMA is a good alternative option of protecting the affected segment of the trachea from manipulation. Moreover, in many studies, P-LMA and ET have been demonstrated to be equally effective in providing adequate ventilation during laparoscopic procedures in adult patients without significant gastric distention or other complications $(8,9)$, while LMA appears to lower the risk of trauma since it is softer and shorter when compared to other airway devices. I-gel has proved to have a role in difficult airway management. It offers more potential advantages such as easy insertion, a short wide airtube and good airway leak pressure (10).

In conclusion, rare congenital anomalies and accompanied focal or long segmental TS can be defined successfully by MSCT before the anesthesia. The management of those difficult airways may be performed using P-LMA and I-gel supraglottic airways and thus avoiding the ET- related complications in some surgical procedures under general anesthesia.

\section{References}

1. Hodina M, Wicky S, Payot M, Sekarski N, Gudinchet F. Non-invasive imaging of the ring-sling complex in children. Pediatr Cardiol 2001; 22: $333-337$.

2. Loukanov T, Sebening C, Springer W, Hagl S. A case of pulmonary artery sling associated with long-segment funnel trachea and bronchus suis. Ann Thorac Surg 2004; 78: 1839-1842.

3. Nagappan R, Parkin G, Wright CA, Walker CS, Vallance N, Buchanan D, Nazaretian S. Adult long-segment tracheal stenosis attributable to complete tracheal rings masquerading as asthma. Crit Care Med 2002; 30: 238-240.

4. Langeron O, Amour J, Vivien B, Aubrun F. Clinical review: Management of difficult airways. Crit Care 2006; 10: 243.

5. Krafft $\mathbf{P}$, Schebesta K. Alternative management techniques for the difficult airway: esophageal-tracheal Combitube. Curr Opin Anaesthesiol 2004; 17: 499-504.

6. Davis DP, Valentine C, Ochs M, Vilke GM, Hoyt DB. The Combitube as a salvage airway device for paramedic rapid sequence intubation. Ann Emerg Med 2003; 42: 697-704.

7. Schragl E, Donner A, Kashanipour A, Gradwohl I, Irich R, Aloy A. Anaesthesia in acute respiratory tract obstructions caused by high degree laryngeal and tracheobronchial stenoses. Anasthesiol Intensivmed Notfallmed Schmerzther 1994; 29: 269-277.

8. Brain AI, Verghese C, Strube PJ. The LMA 'ProSeal' - a laryngeal mask with an oesophageal vent. Br J Anaesth 2000; 84: 650-654.

9. Sinha A, Sharma B, Sood J. ProSeal as an alternative to endotracheal intubation in pediatric laparoscopy. Paediatr Anaesth 2007; 17: 327-332.

10. Joshi NA, Baird M, Cook TM. Use of an i-gel for airway rescue. Anaesthesia 2008; 63: 1020-1021.

Received July 17, 2011. Accepted September 21, 2011. 